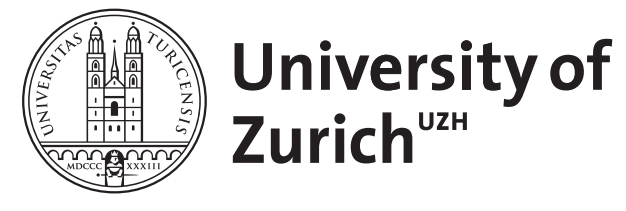

Zurich Open Repository and Archive

University of Zurich

University Library

Strickhofstrasse 39

CH-8057 Zurich

www.zora.uzh.ch

Year: 2008

\title{
Schrift - Text - Bild : Ein Brückenschlag
}

Edited by: Dürscheid, Christa

Posted at the Zurich Open Repository and Archive, University of Zurich

ZORA URL: https://doi.org/10.5167/uzh-15477

Edited Scientific Work

Published Version

Originally published at:

Schrift - Text - Bild : Ein Brückenschlag. Edited by: Dürscheid, Christa (2008). Berlin: de Gruyter. 


\section{Christa Dürscheid}

\section{Schrift - Text - Bild: Ein Brückenschlag}

Texte - denn es sind ja immer Texte, die realisiert und somit auch verschriftlicht werden - werden zuvörderst mit den Augen wahr- und aufgenommen; Texte wirken mithin wie Bilder, wenn nicht gar als Bilder.

Norbert R. Wolf

$(2000,288)$

0. Vorbemerkungen

1. Zum Verhältnis von Schrift, Text und Bild

2. Zur Stellung von Schrift-, Text- und Bildforschung in der Linguistik

3. $\mathrm{Zu}$ den Beiträgen in diesem Heft

4. Literatur

\section{Vorbemerkungen}

Wie der Titel bereits andeutet, will das vorliegende Heft eine Brücke schlagen von der Schrift - genauer: von der Orthographie (siehe den ersten Beitrag von Martin Neef) - zum Bild (siehe den letzten Beitrag von Ulrich Schmitz). Ein solcher Brückenschlag darf aber nicht allein über die Zusammenstellung der Beiträge eines Heftes erfolgen, er muss auch theoretisch gerechtfertigt sein. Deshalb behandelt Abschn. 1 zunächst die Frage, in welcher Relation Schrift und Bild zueinander stehen und wie sich der Text dazu positioniert. Dann wird mit Blick auf die Disziplinen, die das Themenfeld Schrift - Text - Bild umspannen (Graphematik, Orthographie, Textlinguistik, Schreibforschung, Text-Bild-Forschung, Typographie) gezeigt, welchen Stellenwert diese gegenwärtig in der Linguistik haben (Abschn. 2). Abschließend werden die Inhalte der sechs Beiträge dieses Heftes kurz kommentiert.

\section{Zum Verhältnis von Schrift, Text und Bild}

Betrachten wir zunächst die beiden Brückenpfeiler, Schrift und (statisches) Bild. Bei beiden handelt es sich um Ausdrucksformen, die mit Zeichen operieren, die auf Zeichenträgern platziert sind (z.B. Papier, Bildschirm, Stein), die - anders als die gesprochene Sprache - nur mit Hilfe eines Werkzeugs (z.B. eines Schreibgeräts) darstellbar sind und die mit dem Auge wahrge- 
nommen werden. ${ }^{1}$ Folgt man der Klassifikation von Stöckl (2004), dann kann man die Gemeinsamkeiten von Schrift und Bild zeichentheoretisch folgendermaßen erfassen: Sowohl die Schrift als auch das Bild sind „mediale Varianten“. Beide lassen sich je einer „zentralen Zeichenmodalität" (Termini von Stöckl 2004, 17) zuordnen. So ist die Schrift - neben der gesprochenen Sprache - eine mediale Variante der Zeichenmodalität Sprache, das statische Bild - neben dem bewegten Bild - eine mediale Variante der Zeichenmodalität Bild. Während aber im Rahmen der Zeichenmodalität Bild sowohl das statische als auch das bewegte Bild visuell wahrgenommen werden, gilt dies für die komplementäre Zeichenmodalität Sprache nicht: Die geschriebene Sprache wird über das Auge, die gesprochene Sprache über das Ohr erfasst. Es gibt hier also, anders als beim Bild, eine auditive und eine visuelle mediale Variante, und die Frage stellt sich, ob beide Varianten gleichgeordnet sind oder ob die eine der anderen untergeordnet ist (dazu siehe weiter unten). ${ }^{2}$

Neben den Gemeinsamkeiten von Schrift und Bild gibt es auch Unterschiede, die v.a. dann in den Blick kommen, wenn man beide Ausdrucksformen nicht von einer semiotischen, sondern von einer linguistischen Warte aus betrachtet: Das konstitutive Merkmal einer phonographischen Schrift ist, dass ihre diskreten Einheiten, die Grapheme, konventionell auf lautsprachliche Einheiten, auf Phoneme bezogen sind. Diese Graphem-PhonemKorrespondenzen gelten zwar nicht im Sinne einer Eins-zu-Eins-Beziehung, sie legen aber den Rahmen fest, in dem die Schreibung der Lautung zugeordnet wird. So korrespondiert die Graphemsequenz < Ein Buch liegt auf dem Tisch $>$ in der Standardsprache mit einer bestimmten Lautsequenz. Betrachtet man dagegen ein Bild, auf dem eben dieser Sachverhalt dargestellt ist, dann kann man diesen Sachverhalt auf unterschiedliche Weise wiedergeben (z.B. Es liegt ein Buch auf dem Tisch. Auf dem Tisch liegt ein Buch. Ein Buch liegt auf dem Tiscb). Es gibt also keine konventionalisierte lautsprachliche Repräsentation für den im Bild ikonisch dargestellten Sachverhalt. Dies gilt selbst für Piktogramme, die ja häufig anstelle von Schriftzeichen verwendet werden, wenn es darum geht, international verständliche Informationen zu geben (z.B. auf Flughäfen) oder schlicht Platz zu sparen. Beispielsweise hat das Piktogramm $\Varangle$, das sich in der Symbolleiste des Textverarbeitungsprogramms Word findet, zwar eine - für diesen Kontext - festgelegte Bedeu-

1 Dabei ist die Art und Weise, wie das Auge die Sehfläche beim Lesen und beim Betrachten von Bildern abtastet, vergleichbar (vgl. Kress/van Leeuwen 1996). Die Autoren sprechen denn auch von "Reading images" und verbinden damit das Dekodieren von Geschriebenem und das Betrachten von Bildern.

2 Bekanntlich existiert noch eine weitere mediale Variante von Sprache, die ebenfalls visuell wahrgenommen wird: die gebärdete Sprache. Diese wird in der Klassifikation von Stöckl (2004, 17) nicht erwähnt. Er nennt zwar die Gestik, die Körperhaltung und die Mimik als "Submodalitäten“. Dass er in diesem Zusammenhang aber nicht die Gebärdensprache im Blick hat, zeigt sich daran, dass er diese Submodalitäten als nonverbal klassifiziert. 
tung, diese Bedeutung kann aber unterschiedlich versprachlicht werden (z.B. Den markierten Text ausschneiden. Den markierten Text entfermen. Den markierten Text löschen).

Ein weiterer Unterschied zwischen Schrift und Bild ist zeichentheoretischer Art: Für jedes Schriftsystem gilt, dass es mit symbolischen Zeichen operiert. Diese Aussage trifft sowohl für den phonographischen Schrifttyp zu, zu dem das deutsche Schriftsystem gehört, als auch für den logographischen Schriftyp des Chinesischen. ${ }^{3}$ Es gibt also keine Ähnlichkeitsbeziehung zwischen den Zeichen und dem, was die Zeichen in ihrer Kombination bedeuten. Anders ist es bei Bildern: Bilder können sich ikonisch auf Gegenstände beziehen oder abstrakte Darstellungen sein. Freilich können Bilder in ihrer gegenständlichen bzw. abstrakten Darstellung auch symbolischen Charakter haben (wie z.B. das Bild einer Taube auf einem Stadtwappen oder ein handgemaltes Herz auf Briefpapier). Doch ist diese symbolische Lesart, anders als bei der Schrift, nur eine von mehreren Möglichkeiten der Interpretation von Bildern. Wichtig ist in diesem Zusammenhang auch, dass Schriftzeichen, anders als Bilder, nur in der Verknüpfung miteinander, nicht aber isoliert Bedeutung tragen. So hat zwar die Graphemsequenz < gut > eine Bedeutung, nicht aber die Grapheme $\langle\mathrm{g}\rangle,\langle\mathrm{u}\rangle$ und $\langle\mathrm{t}\rangle$. Diese stehen, wie auch Phoneme, nur für eine Ausdrucksseite, nicht für eine Inhaltsseite. ${ }^{4}$

Aus dem Umstand, dass die Schrift auf symbolischen Zeichen aufbaut, folgt ein weiterer Unterschied zum Bild: Es gibt Regeln zur linearen Verknüpfung der Schriftzeichen. Diese werden in der Graphematik beschrieben und in der Orthographie festgeschrieben. Für Bilder dagegen gibt es solch normative Regeln nicht. Ulrich Schmitz $(2006,190)$ sagt dies deutlich:

Entsprechend können Bilder zwar gut oder schlecht sein und bestimmten ästhetischen Vorstellungen oder stilistischen Konventionen mehr oder wenig gut entsprechen. Sie können aber nicht richtig oder falsch gebaut sein oder gegen verbindlich anerkannte Regeln verstoßen [...].

Damit kommen wir zum nächsten Punkt: In welchem Verhältnis steht der Text zur Schrift und zum Bild? Wie das vorangestellte Zitat von Norbert R. Wolf nahe legt, besteht der Text einerseits aus Schriftzeichen, hat andererseits aber auch Bildcharakter. Betrachten wir zunächst den ersten Teil dieser Aussage. Wer sagt, Texte bestehen aus Schriftzeichen, setzt voraus, dass Texte schriftlich fixierte Äußerungen sind. Eine solche Auffassung ist keineswegs unumstritten. Man kann sich durchaus auf den Standpunkt stellen,

3 Wenn auch unter bestimmten Vorbehalten (vgl. Dürscheid 2006, 68-78). So gibt es im Chinesischen Schriftzeichen, deren bildhafter Charakter noch erkennbar ist.

4 Das Gesagte gilt selbstverständlich auch für Abkürzungsschreibungen. Beispielsweise hat das Zeichen $<P>$ auf einem Parkplatzschild keine Bedeutung; es steht lediglich als Kürzel für eine Graphemkombination (z.B. für die Sequenz <Parkplatz $>$ ), die ihrerseits Bedeutung trägt. 
dass auch mündliche Äußerungen unter spezifischen Bedingungen Texte sind (vgl. zu einer Diskussion dieser Frage Adamzik 2004, 41-43; siehe auch Dürscheid 2007) - und zwar insbesondere dann, wenn es sich um monologische Sequenzen handelt (z.B. Vorträge, Predigten, politische Reden, Referate). Das ändert aber nichts an dem Umstand, dass Texte schriftlich vorkommen können und dass dies zweifellos das prototypische Vorkommen von Texten ist. Wenn hier also Text in eine Reihe mit Schrift und Bild gestellt wird, dann ist dies deshalb gerechtfertigt, weil von einer Subklasse von Texten, von schriftbasierten Texten ausgegangen wird.

Eine weitere wichtige Gemeinsamkeit zwischen Schrift, Text und Bild ist: Ihr Ordnungsprinzip ist die Fläche. Während dies bei (schriftbasierten) Texten und (statischen) Bildern unmittelbar einleuchtet, kann man einwenden, dass die Schrift, anders als der Text, eindimensional, nicht zweidimensional angeordnet ist. Gegen eine solche Auffassung wendet sich Sybille Krämer aus sprachphilosophischer Sicht. Sie vertritt in mehreren ihrer Arbeiten dezidiert den Standpunkt, dass die Schrift sich keineswegs auf die Verschriftung gesprochener Sprache reduzieren lasse, sondern ein eigenständiges Symbolsystem darstelle, das von der Zweidimensionalität der Fläche Gebrauch mache (vgl. Krämer 2004, 158-160). So sei gerade die „Zwischenräumlichkeit" ein konstitutives Strukturprinzip, das dazu führe, dass grammatische Strukturen überhaupt wahrgenommen würden. ${ }^{5}$ Krämer verweist auf Untersuchungen aus der Leseforschung, die gezeigt haben, dass Texte nicht sequentiell-linear, von links nach rechts gelesen werden, sondern als Fläche wahrgenommen werden, auf der das Auge von einem Sehpunkt zum anderen springt. Schrift ist also, wenn man Krämer folgt, mehr als die lineare Anordnung von Buchstaben, Schrift ist gestaltete Fläche. Diese Auffassung wurde in der Linguistik lange Zeit ausgeblendet; erst in neueren Arbeiten zur Typographie wurde diesem Umstand Rechnung getragen (vgl. zu einem Überblick Spitzmüller 2006).

Allerdings muss festgehalten werden, dass Krämers Kritik an dem - von ihr so genannten - „Linearitätsdogma“ (vgl. 2004, 159) nur dann berechtigt ist, wenn man das Entstehen des Textes auf der Makroebene, als Ganzes, betrachtet und wenn man, wie sie es tut, auf die Rezipientenperspektive Bezug nimmt. Zunächst zum letztgenannten Punkt: Beim Lesen ist die zweidimensionale Ordnung in der Tat die relevante Größe; das Lesen erfolgt, wie weiter oben schon gesagt wurde, nicht linear, nicht Buchstabe für Buchstabe, nicht Wort für Wort, nicht von links nach rechts (vgl. dazu ausführlich Gross 1994). Das gilt auch für den Schreiber, der ja immer auch ein Leser seines eigenen Textes ist. Auch als Textproduzent orientiert sich der Schreiber an der Fläche, so z.B. wenn er Absätze vorsieht, einen bestimmten

5 In der Tat: Man versuche z.B., die Bedeutung des Wortes Wort ohne Rückgriff auf die Schrift zu erfassen. 
Blattrand wählt, Leerzeilen einfügt, den Text in Spalten anordnet. Dieser Bereich der Textgestaltung erfolgt also in der Tat nicht linear, sondern unterliegt typographischen Gestaltungsprinzipien, die auf die Fläche Bezug nehmen. $\mathrm{Zu}$ den Textgestaltungsprinzipien gehören aber auch solche, die sich auf den Zeichen- bzw. Wortabstand beziehen (vgl. Stöckl 2004, 22) und damit auf die lineare Anordnung der Zeichen. Außerdem ist es auch die Schreibtätigkeit selbst, das Buchstabenschreiben (bzw. das Buchstabentippen), das eindimensional erfolgt, denn die Schriftzeichen werden im Deutschen linear, nicht flächig angeordnet. ${ }^{6}$ Es ist also keineswegs so, dass nur die Fläche eine relevante Bezugsgröße ist; auch die lineare Dimension ist beim Schreiben wichtig. Krämer hat aber Recht, wenn sie feststellt, dass die lineare Dimension bislang die zentrale Rolle in der linguistischen Analyse spielte.

Wie wir an dieser Diskussion zum Schreiben sehen, umfasst der Terminus Schreiben mehrere Bedeutungen, vom basalen Buchstabenschreiben bis zum Verfassen von Texten und dem Anfertigen von Skripten (siehe Ludwig in diesem Heft). Es muss also immer auch die Frage geklärt werden, auf welches Konzept von Schreiben man Bezug nimmt, wenn man vom Schreibprozess spricht. Verstehen wir unter Schreiben das Verfassen von Texten, so gilt für dieses Schreiben selbstverständlich nicht, dass es linear erfolgt - und zwar weder in der räumlichen noch in der zeitlichen Dimension. Die Textproduktion ist vielmehr ein komplexer Vorgang, der mehrere Phasen durchläuft, die mehrfach in sich verschränkt sein können. Es sind dies das Planen, das Formulieren und Revidieren des Textes (vgl. dazu das in der Schreibforschung viel diskutierte Schreibprozessmodell von Hayes/Flower 1980). Diese drei Phasen gelten bis heute als konstitutiv, auch wenn das Modell als Ganzes mehrfach modifiziert wurde (vgl. Sieber 2003).

Den Punkt abschließend sei noch ein Wort zum Verhältnis von Text und Bild gesagt (vgl. dazu ausführlich Fix/Wellmann 2000): Bilder können in Texte integriert sein (z.B. als Fotos in einem Zeitungsartikel oder als Diagramme in einer wissenschaftlichen Abhandlung). Bilder können aber auch neben Texten stehen, wie dies bei einer MMS-Botschaft über das Handy der Fall ist, wenn das Bild um einen schriftlichen Kommentar ergänzt wird. Weiter kann man den Standpunkt vertreten, dass Texte allein aufgrund ihres Layouts Bildcharakter haben. Dieses ,Textbild' stellt eine eigene, konnotative Bedeutungsebene dar, die den Text gewissermaßen überlagert. Der Text ist also, um mit Schmitz $(2006,194)$ zu sprechen, eine „Sehfläche“, die typo-

6 Dies gilt z.B. nicht für das koreanische Schriftsystem, wo die Schriftzeichen flächig, in kleinen Quadraten erscheinen, die jeweils für eine Silbe stehen (vgl. Dürscheid 2006, 87-95). 
graphisch gestaltet ist. ${ }^{7}$ Dabei ist es nicht nur der Text als Ganzes, der über Absätze, Einrückungen und das Einbinden von Grafiken usw. gestaltet wird, zum Textdesign gehört auch die Gestaltung der Schrift (z.B. im Hinblick auf Schriftart, Schriftgröße, Schriftfarbe) ${ }^{8}$ Stöckl $(2004,22)$ spricht in diesem Zusammenhang von der Makro-, der Meso- und der Mikrotypographie.

Wichtig ist an dieser Stelle noch der Hinweis, dass der Text als Analysekategorie in der Klassifikation Stöckls, die zentrale und periphere Zeichenmodalitäten, mediale Varianten und korrespondierende Gestaltungsmöglichkeiten umfasst, gar nicht vorkommt. Das überrascht nicht: Der Text stellt nicht selbst eine Zeichenmodalität dar, er ist vielmehr das Resultat des Zusammenspiels von Zeichenmodalitäten. Stöckl bezeichnet ihn deshalb als "multimodale Einheit". Auch in dieser Hinsicht ist also der Brückenschlag von der Schrift zum Bild gar nicht so groß: Der Text vereint nach dieser Auffassung alle Zeichenmodalitäten in sich - und damit auch Schrift und Bild.

\section{Zur Stellung von Schrift-, Text- und Bildforschung in der Linguistik}

Die vorangehenden Ausführungen haben auf mehrere linguistische Disziplinen Bezug genommen, auf die Graphematik, die Orthographie, die Textlinguistik, die Schreibforschung, die Text-Bild-Forschung und die Typographie. Dabei handelt es sich um Forschungsfelder, von denen einige noch vor zehn Jahren in der Linguistik kaum eine Rolle spielten. Dies gilt insbesondere für die Graphematik, die Orthographie und die Typographie, in Ansätzen auch für die Text-Bild-Forschung. Die Textlinguistik und die Schreibforschung haben dagegen schon eine etwas längere Tradition. Die Textlinguistik etablierte sich im deutschsprachigen Raum bereits in den 1960er-Jahren (vgl. Adamzik 2004), die Schreibforschung Ende der 1980er-Jahre (vgl. Ludwig 2001). ${ }^{9}$ Auch das Verhältnis von Text und Bild ist schon länger Gegenstand

7 Nota bene: Schmitz bezieht den Terminus ${ }_{n}$ Sehfläche ${ }^{\star}$ nicht auf Printseiten, sondern auf Bildschirmseiten im Internet. Er stellt dazu fest: „Auf den obersten Ebenen der meisten Internetangebote erblickt man ein Sammelsurium von Bildern, Texten, Wörtern, Logos und visuellen Zeichen auch jeglicher anderer Art" (Schmitz 2006, 193).

8 Vgl. zu den verschiedenen Aspekten des Textdesigns auch den Sammelband von Roth/ Spitzmüller (2007), in dem mit Blick auf verschiedene Anwendungsdomänen gezeigt wird, in welcher Wechselwirkung Textdesign und Textinhalt stehen.

9 Ziel der Schreibforschung ist es, zu empirisch abgestützten Befunden über Schreibprozesse zu gelangen und diese theoretisch aufzuarbeiten. An die Schreibforschung knüpft die Schreibdidaktik an, die u.a. der Frage nachgeht, wie die Schreiblkompetenz gefördert werden kann. Diese Frage ist insbesondere für die Deutschdidaktik relevant. So enthält das neueste Heft der Deutschdidaktikzeitschrift ide, das den Titel „Kultur des Schreibens“ trägt (Heft 1/2007), mehrere Beiträge zur Entwicklung der Schreibkompetenz (vgl. auch Brommer in diesem Heft). 
linguistischer Forschung, doch wurden beide in der Regel als interagierende, aber separate Einheiten betrachtet. Das ist in der neueren Forschung, in der man Texte als multimodale Einheiten ansieht (vgl. Stöckl 2004), anders. Damit verbunden ist, dass die traditionelle textlinguistische Auffassung, nur sprachliche Gebilde seien Texte, aufgegeben werden muss. ${ }^{10}$

Was nun die Graphematik, die Orthographie und die Typographie betrifft, so gilt, dass die damit verbundenen Forschungsfelder erst in jüngster Zeit in der Linguistik Beachtung finden. Dass sich hier ein Wandel abzeichnet, sei eigens betont, denn außerhalb der Linguistik herrscht meist noch die Auffassung vor, der Schrift komme in unserer Disziplin keine Relevanz zu (vgl. z.B. Krämer 2004), sie werde lediglich, wie es seit dem berühmten Diktum von Ferdinand de Saussure heißt, als ein sekundäres, der gesprochenen Sprache nachgeordnetes System angesehen. Eine solch logoszentrierte Auffassung wird in der Linguistik als Dependenzhypothese bezeichnet. In Kontrast dazu steht die Autonomiehypothese. Die Vertreter dieser Position machen geltend, dass die Schrift eine eigenständige Realisationsform von Sprache sei, die theoretisch und methodisch von der gesprochenen Sprache zu unterscheiden ist (vgl. Dürscheid 2006). Dass diese Auffassung immer mehr an Boden gewinnt, zeigt sich an den linguistischen Publikationen, die in jüngster Zeit in diesem Bereich zu verzeichnen sind. Sie seien hier nicht im Einzelnen aufgeführt, denn auf einige wird in den folgenden Beiträgen ohnehin Bezug genommen. Ergänzend dazu möchte ich an dieser Stelle aber vier Punkte festhalten, die mir interessant scheinen, weil sie zeigen, dass schriftlinguistische Themen sowohl für die Forschung als auch für die Lehre relevant geworden sind:

1) Die Zeitschrift für Sprachwissenschaft, das Publikationsorgan der „Deutschen Gesellschaft für Sprachwissenschaft" (DGfS), widmet ihr Jubiläumsheft 2007 dem Thema "Orthographie und Sprachwissenschaft". Im Vorwort wenden sich die Herausgeber explizit gegen die (lange Zeit vorherrschende) Marginalisierung der Orthographie. Sie schreiben: „Orthographie könnte [...] denjenigen linguistischen Disziplinen neue Erkenntnismöglichkeiten bieten, die sie mit dem Argument ihrer theoretischen Bedeutungslosigkeit aus ihren Betrachtungen ausschließen" $(2007,1)$.

2) Im Rahmen eines großen, auf 25 Bände geplanten Wörterbuchprojekts ist ein Fachwörterbuch zur Schriftlinguistik in Arbeit, das von Rüdiger Weingarten und Martin Neef herausgegeben wird (Bd. 5). Dieses Wörterbuch steht in einer Reihe neben anderen im Projekt geplanten Wörterbüchern zu etablierten Gebieten der Linguistik (z.B. Phonetik und Phonologie, Grammatik, Wortbildung). Weitere Informationen dazu geben die

$\overline{10}$ Siehe dazu aber die Ausführungen von Kirsten Adamzik $(2004,43)$, die sich für einen auf Sprachliches eingegrenzten Textbegriff ausspricht. 
beiden Reihenherausgeber, Stefan J. Schierholz und Herbert Ernst Wiegand, in diesem Heft.

3) Eine neue linguistische Fachzeitschrift, Written Language and Literacy, widmet sich ausschließlich der Struktur und Funktion geschriebener Sprache. Das Themenspektrum dieser Zeitschrift ist vielfältig. Dies geht bereits aus der Kurzbeschreibung des Benjamins-Verlages hervor, bei dem die Zeitschrift erscheint (siehe unter http://www.benjamins.com). Daraus ein Auszug: "Written Language and Literacy will be concerned with (neuro)linguistic, psycholinguistic, educational and sociolinguistic accounts of the structure and functions of written language, the processes and acquisition of reading and writing, and the use and development of literacy in different social and cultural settings."

4) Linguistische Einführungswerke behandeln zunehmend schriftlinguistische Themen (z.B. Orthographie/Graphematik). Während dies noch vor einigen Jahren v.a. dann der Fall war, wenn sich die Einführung an Lehramtsstudierende richtete, gibt es nun auch in fachwissenschaftlich ausgerichteten Einführungen entsprechende Kapitel. So erschien vor kurzem ein Studienbuch Germanistische Linguistik (Busch/Stenschke 2007) mit einem Kapi-

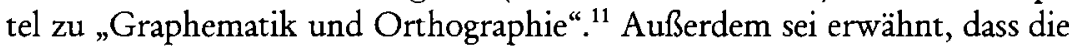
Einfübrung in die Schriftlinguistik (Dürscheid 2006) in ihrer dritten Auflage um ein Kapitel zur Typographie von Jürgen Spitzmüller ergänzt wurde.

Wie die vier genannten Aspekte zeigen, gibt es nun in der Linguistik eine Herangehensweise an das Thema Schrift und Schreiben aus verschiedenen Richtungen. Auch das vorliegende Heft steht in diesem Kontext. Noch haben allerdings die meisten Ansätze strukturbeschreibenden Charakter; soziolinguistische Analysen fehlen weitgehend (vgl. aber Spitzmüller in diesem Heft). Das hängt sicher damit zusammen, dass die Schreibung lange Zeit als "das am wenigsten variable Teilsystem von Sprache betrachtet wurde“ (Androutsopoulos 2007, 93). Diese Ära scheint nun, wie Androutsopoulos weiter schreibt, vorbei zu sein. Als Evidenz lässt sich anführen, dass durch die Nutzung der neuen Medien ein immer breiteres Spektrum an schriftlichen Äußerungsformen im Schreiben entsteht, aber auch, dass das Schriftbild heutzutage von Laien vielfach variiert werden kann. Androutsopoulos $(2007,93)$ beschreibt die Veränderungen zusammenfassend sehr treffend:

11 Die Graphematik wird in dieser Einführung in einem umfassenden Sinne als Teildisziplin der Linguistik verstanden, die "sich mit der Schreibung von Wörtern und deren Zustandekommen befasst" (Busch/Stenschke 2007, 58), die Orthographie als ein „explizit geregeltes, konventionalisiertes System von Normen“ (ebd. 59). Martin Neef (2005) legt dagegen eine enge Definition von Graphematik zugrunde, wonach diese ausschließlich die Beziehung zwischen schriftlichen Formen und deren phonologischen Repräsentationen erfasst (siehe auch Neef in diesem Heft). 
Überhaupt sind die gegenwärtigen Wandelprozesse vielleicht nirgendwo sonst so deutlich wie in der Schreibung, da sie der Repräsentation sprechsprachlicher Muster genauso dient wie einer von der Phonie unabhängigen, expressiven bzw. sozialsymbolischen Manipulation des Schriftbildes.

Es gibt also noch weitere, über die Strukturbeschreibung hinausgehende Fragen, die in der Schriftlinguistik zu bearbeiten sind - und dies auch deshalb, weil der Untersuchungsgegenstand selbst, die Schrift, eine noch nie dagewesene Dynamik zeigt. Die folgenden zwei Desiderata möchte ich abschließend festhalten:

1) Die derzeit zu beobachtenden Umbrüche im Schreiben resultieren zum einen aus den typographischen Möglichkeiten, über die heute jedes Textverarbeitungssystem verfügt, zum anderen aus der quasi-synchronen Kommunikation, wie sie seit einigen Jahren im Geschriebenen möglich ist. Die Veränderungen betreffen also sowohl die visuelle als auch die konzeptionelle Ebene. Beiden Ebenen muss man in der linguistischen Analyse Rechnung tragen.

2) Will man schriftbasierte Phänomene vollständig erfassen, dann genügt es nicht, sie aus systemlinguistischer Sicht zu beschreiben. Man muss auch die Frage stellen, wie sich die Schrift(lichkeit) in der sozialen Interaktion gestaltet und welche kommunikativen Funktionen damit verbunden sind. Es ist also ein weiterer Brückenschlag - von der systemlinguistischen zur soziolinguistischen Analyse - erforderlich.

\section{Zu den Beiträgen in diesem Heft}

Wie schon erwähnt, umfasst der thematische Teil des vorliegenden Heftes sechs Beiträge, die in ihrer Abfolge den Bogen von der Schrift zum Bild spannen. Den Auftakt macht Martin Neef mit der Analyse eines Regelausschnitts aus der deutschen Orthographie. Als Untersuchungsgegenstand wählt Neef den Regelbereich "Worttrennung am Zeilenende“. Dieser ist sowohl in der Amtlichen Regelung als auch über die Einzelfallfestlegungen in den Wörterbüchern kodifiziert. In seiner Analyse knüpft Neef an das graphematische Rekodierungsmodell an, das sehr systematisch die Übersetzung von schriftlichen Formen in phonologische Repräsentationen beschreibt (vgl. Neef 2005). ${ }^{12}$ Dieses Modell hat, wie er darlegt, einen linearen, segmentalen Charakter; die Regularitäten werden bevorzugt über die Abfol-

12 Hier muss nochmals angemerkt werden, dass Neef einen engen Graphematikbegriff zugrunde legt. Bestimmte Regelbereiche, wie z.B. die Groß- und Kleinschreibung und die Getrennt- und Zusammenschreibung, können diesem Konzept zufolge gar nicht innerhalb der Graphematik beschrieben werden. Was die Worttrennung am Zeilenende betriff, so ist dies aber keineswegs ausgeschlossen. Wie Neef denn auch zeigt, sind die Trennungsregularitäten primär graphematisch motiviert. 
ge der Buchstaben analysiert. Als Ausgangspunkt dient ihm also das eindimensionale Ordnungsprinzip der Schrift; die Fläche spielt in seinem Modell keine Rolle (vgl. die obige Diskussion).

Ziel von Neefs Beitrag ist es, die Worttrennungsdaten, wie sie den Wörterbüchern zu entnehmen sind, in einer linguistischen Rekonstruktion zu erfassen. Als Orientierungsrahmen dienen ihm die einschlägigen Paragraphen der Amtlichen Regelung. Diese bestehen aus einer Grundregel ( $\$ 107)$, nach der die Silbengrenzen beim lauten Lesen mit den Trennstellen beim Schreiben übereinstimmen. Hinzu kommen drei weitere Trennungsregeln, von denen eine auf die morphologische Struktur von Wörtern Bezug nimmt, die anderen beiden auf das Auftreten von Vokal- bzw. Konsonantenbuchstaben. Neef formuliert in kritischer Auseinandersetzung mit diesen Regeln eine Reihe von Trennungsbeschränkungen, die die Worttrennungsdaten erklären sollen. Ob ihm dies in allen Fällen gelungen ist, ließe sich nur sagen, wenn alle Einzelschreibungen im Wörterbuch überprüft würden, was natürlich im vorliegenden Rahmen nicht zu leisten ist. Im Fazit ergibt sich, dass die Worttrennung am Zeilenende primär auf den Buchstaben als zentrale Bezugseinheit basiert. Erst in einem zweiten Schritt wird diese Grundregularität von einem silbenbasierten Konzept überlagert, das aber, so betont Neef abschließend, „in Kernbereichen des Regelungsgegenstands nicht anwendbar ist".

Auch der zweite Beitrag nimmt Bezug auf die Orthographie; hier geht es aber nicht um die Analyse der dahinter stehenden Systematik, sondern um die Frage, welche Meinungen über die Rechtschreibleistungen der Jugendlichen in der Öffentlichkeit vorherrschen. Denn wie Sarah Brommer in ihrer empirischen Studie zur Schreibkompetenz der Jugendlichen im öffentlichen Diskurs zeigt, sind es insbesondere die Rechtschreibfähigkeiten, die in der Öffentlichkeit immer wieder thematisiert werden. Ihr Beitrag stützt sich auf die Auswertung von 671 Äußerungen in 216 Zeitungs- und Zeitschriftenartikeln aus den Jahren 1994 bis 2005. Die in diesen Artikeln vorgebrachten Meinungen zur Schreibkompetenz werden kritisch analysiert, die dahinter stehenden Argumentationsstrukturen aufgedeckt und Verschränkungen mit verwandten Diskursen (z.B. zur Bildungspolitik) betrachtet. Ein besonderes Verdienst des Beitrags ist, dass der Terminus Schreibkompetenz operationalisiert wird, genauer: dass zwischen einem formalen und einem stilistischen Bereich unterschieden wird. Schreibkompetenz im formalen Bereich bezieht sich auf die formale Sprachrichtigkeit (was Brommer als ,Schreibfähigkeit' bezeichnet), Schreibkompetenz im stilistischen Bereich auf das stilistisch angemessene, logisch-stringente Schreiben (was Brommer als ,Ausdrucksfähigkeit' bezeichnet).

Ihre Datenanalyse macht deutlich, dass die Schreibkompetenz zumeist kritisch beurteilt und als unzureichend eingeschätzt wird. Allerdings wird 
die Frage, welche Bereiche der Schreibkompetenz gemeint sind, in den meisten Artikeln nicht geklärt. Wie die inhaltliche Auswertung aber zeigt, ist es v.a. die Schreibfähigkeit, die kritisch gesehen wird, wobei gerade in diesem Bereich die Äußerungen oft sehr emotional sind. Andere Fähigkeiten (z.B. wie die Fähigkeit, einen kohärenten Text zu verfassen) stehen dagegen weniger im Fokus. Hier wird die Auseinandersetzung zudem weniger emotional geführt; sachbezogene Stellungnahmen überwiegen.

Der dritte Beitrag, verfasst von Peter Koch und Wulf Oesterreicher, trägt den Titel „Schriftlichkeit und kommunikative Distanz“. In ihrem Beitrag nehmen die beiden Autoren Bezug auf frühere gemeinsame Arbeiten zu medialer resp. konzeptioneller Mündlichkeit und Schriftlichkeit (z.B. Koch/ Oesterreicher 1994). Die in diesen Publikationen vorgetragenen Überlegungen wurden in der Fachwelt unter der Bezeichnung "das Modell von Koch/Oesterreicher" bekannt. In diesem Modell wird dargestellt, in welcher Relation konzeptionelle Mündlichkeit und Schriftlichkeit bzw. kommunikative Nähe und kommunikative Distanz zueinander stehen und über welche Parameter diese beiden Pole kommunikativen Handelns beschrieben werden können. Es wird von einem universalen, einzelsprachübergreifenden Kontinuum zwischen Nähe und Distanz ausgegangen, einer Variabilität also, die plausibel macht, dass dieser Ansatz, wie die Autoren schreiben, ,immer auch ein Beitrag zur Varietätenlinguistik" ist. Interessant ist nun ihre These, dass die drei Dimensionen Diatopik, Diastratik, Diaphasik, die in der Varietätenlinguistik als konstitutiv für Sprachvariation angesehen werden, von dem Kontinuum zwischen Nähe und Distanz überlagert werden. Dieses Kontinuum ist, so Koch/Oesterreicher, eine anthropologische Konstante, die in allen Sprachgemeinschaften zu jedem Zeitpunkt gegeben ist.

Damit haben die Autoren die theoretische Grundlage geschaffen, um in einem zweiten Schritt die Leistungsfähigkeit des Modells aufzuzeigen und es an aktuelle Forschungen anzuknüpfen. In diesem Zusammenhang wird auch kurz auf neuere Arbeiten aus der Medienlinguistik eingegangen, die die Anwendbarkeit des Modells auf die E-Mail- und Chat-Kommunikation diskutieren. Interessant sind auch die Ausführungen zur Relevanz, die das NäheDistanz-Kontinuum für soziolinguistische Fragestellungen (z.B. die Beschreibung von Mehrsprachigkeit) hat. Und schließlich wird dafür argumentiert, dass das Modell "nicht allein für die Synchronie sprachlicher Situationen gültig ist", sondern auch diachronische Aspekte erfassen kann.

Kommen wir zum nächsten Beitrag von Otto Ludwig, „Skripte. Konturen einer Konzeption", der die Unterscheidung von Text und Skript ins Zentrum seiner Überlegungen stellt. Wie Ludwig deutlich macht, müssen die beiden Termini Text und Skript sorgfältig unterschieden werden: Skripte sind Niederschriften von Texten (als Manu-, Typo- oder Compuskripte), sie stellen aber nicht selbst Texte dar. Texte entstehen, so Ludwig, im Kopf des 
Produzenten, und sie werden rekonstruiert im Kopf des Rezipienten. Dazwischen liegt ein dritter Zustand von Text, der zeitlich gesehen der zweite ist: Texte werden niedergeschrieben; sie werden auf einen materiellen Träger, dem Skript, platziert, das „ihnen Dauer und damit Überlieferungsqualitäten verleiht." Vorgestellt werden die wichtigsten kategorialen Einheiten von Skripten (z.B. Zeile, Seite), wobei Ludwig u.a. an der Unterscheidung von Absätzen (als skriptbezogene Einheiten) und Abschnitten (als textbezogene Einheiten) deutlich macht, wie die inhaltliche Struktur von Texten in der Gestaltung von Skripten gespiegelt wird.

Zum Schluss seines Beitrags präsentiert Ludwig die theoretische Einordnung seiner Überlegungen. Er spricht sich für einen handlungstheoretischen Ansatz aus, in dem die Produktion von Skripten in einer Reihe neben anderen Tätigkeiten steht, d.h. neben der Handlung der Buchstabenproduktion, der Wortproduktion, der Satzproduktion und der Textproduktion. Eine solche Unterscheidung verschiedener Handlungen, die beim Schreiben relevant sind, ist für die Schreibforschung von zentraler Bedeutung. Zudem kann mit der von Ludwig vorgenommenen Unterscheidung von Skript und Text der jeweilige Bezugsrahmen, in dem schriftlinguistische Untersuchungen durchgeführt werden, klar umrissen werden. So lässt sich festhalten, dass typographisch ausgerichtete Studien, wie z.B. die Arbeit von Stöckl (2004), auf Skripte, nicht auf Texte Bezug nehmen. Streng genommen kann also auch nicht von multimodalen Texten die Rede sein; in Ludwigs Sinne müsste man von multimodalen Skripten sprechen.

Auch Jürgen Spitzmuillers Beitrag mit dem Titel "Graphisches Crossing“ ist eine solch typographisch ausgerichtete Studie. Im Zentrum steht hier die Frage, wie soziolinguistische Methoden für die Analyse graphostilistischer Variation eingesetzt werden können. Als Ausgangspunkt dient Spitzmüller das Konzept des Language Crossing. Darunter wird der Wechsel von einem vertrauten in einen dem Sprecher fremden Code verstanden - im Gegensatz zum Code Switcbing, wo es sich um einen Wechsel zwischen mehreren, dem Sprecher vertrauten Sprachen oder Varietäten handelt. Dieses CrossingKonzept überträgt Spitzmüller auf die Alternation graphischer Zeichensysteme. Er definiert graphisches Crossing als „die Alternation mehrerer graphischer Systeme bzw. Subsysteme, von denen mindestens eines von den Interaktionspartnern als dem Produzenten ,fremd" erachtet wird." Im Anschluss daran werden drei Beispiele für ein solches Crossing vorgestellt: Crossing im Bereich der Graphematik, Crossing im Bereich der Typographie und Crossing zwischen verschiedenen Schriftarten und Schrifttypen.

Spitzmüllers Analysen machen deutlich, dass graphostilistische Variation weit mehr ist als das gezielte Durchbrechen von Normen mit dem Ziel, Aufmerksamkeit zu erlangen (wie z.B. in der Werbung). Vielmehr stellt aus seiner Sicht die als ,fremd' wahrgenommene Verwendung graphischer Ele- 
mente einen wichtigen Bestandteil der Identitätsarbeit innerhalb sozialer Gruppen dar. Die Grenzübertretungen sind also ein konstitutives Element kommunikativer Praxis. Sie dienen "als Kontextualisierungshinweise, die gemeinsames Wissen evozieren und dadurch den Interpretationsrahmen eines Textes abstecken." Zum Schluss seiner Ausführungen plädiert Spitzmüller nachdrücklich dafür, soziolinguistische Methoden in die Schriftlinguistik einzubeziehen - ein Plädoyer, dem ich mich nur anschließen kann.

Damit komme ich zum letzten Beitrag in der Themenreihe, übertitelt mit "Bildakte? How to do things with pictures“. In diesem legt Ulrich Scbmitz dar, dass es zwar auf semantischer Ebene charakteristische Unterschiede zwischen Bildern und Sprache gebe, dass aus pragmatischer Sicht aber wichtige Gemeinsamkeiten bestehen. Er vertritt die Auffassung, dass die Sprechakttheorie Austins und Searles auch auf Bilder Anwendung finden kann. Um dies zu illustrieren, analysiert er eine Plakat-Kampagne der Autofirma Toyota, die aus zwanzig verschiedenen Motiven besteht, und weist Schritt für Schritt nach, dass sich alle fünf Sprechaktklassen in diesen Plakaten wiederfinden.

Führt man Schmitz' Überlegungen weiter - und er deutet das zum Schluss nur an -, dann bietet es sich an, „die vielfältigen Bildsorten mit ihren unterschiedlichsten Ausprägungen von Bildgebrauch genauer unter die Lu$\mathrm{pe}^{\text {" }} \mathrm{zu}$ nehmen. Man könnte also versuchen, verschiedene Typen von Bildkommunikation mit dem Instrumentarium der Sprechakttheorie zu beschreiben. $\mathrm{Ob}$ dies möglich ist, wird von Schmitz nicht diskutiert, er beschränkt sich auf eine Plakatanalyse. In dieser Analyse geht er aber so systematisch vor, dass man die im Titel angedeutete Frage, ob es sinnvoll sei, von „Bildakten“ zu sprechen, bejahen möchte.

\section{Literatur}

Adamzik, Kirsten (2004): Textlinguistik. Eine einführende Darstellung. Tübingen: Niemeyer (= Germanistische Arbeitshefte 40).

Androutsopoulos, Jannis (2007): Neue Medien - neue Schriftlichkeit? In: Mitteilungen des Germanistenverbandes. 54, H. 1. Thema des Heftes: Medialität und Sprache, S. 72-97.

Busch, Albert/Stenschke, Oliver (2007): Germanistische Linguistik. Eine Einführung. Tübingen: Narr (= bachelor wissen).

Deutschunterricht. Thema des Heftes: Standards: Schreibkompetenz. 60, H. 1 (2007).

Dürscheid, Christa (2006): Einführung in die Schriftlinguistik. Ergänzt um ein Kapitel zur Typographie von Jürgen Spitzmüller. 3., überarbeitete und ergänzte Auflage. Göttingen: Vandenhoeck \& Ruprecht.

Dürscheid, Christa (2007): Texte aus kommunikativ-pragmatischer Sicht. In: Zeitschrift für Angewandte Linguistik. 46, H. 1, S. 3-18.

Fix, Ulla/Wellmann, Hans (Hrsg.) (2000): Bild im Text - Text im Bild. Heidelberg: Winter (= Sprache - Literatur und Geschichte 20). 
Gross, Sabine (1994): Lese-Zeichen. Kognition, Medium und Materialität im Leseprozeß. Darmstadt: Wissenschaftliche Buchgesellschaft.

Hayes, John R./Flower, Linda (1980): Identifying the Organization of Writing Processes. In: Gregg, L. W./Steinberg, E. R. (Hrsg.) (1980): Cognitive Processes in Writing. Hillsdale: Lawrence Erlbaum Ass., S. 3-30.

ide. Informationen zur Deutschdidaktik. Zeitschrift für Deutschunterricht in Wissenschaft und Schule. Thema des Heftes: Kultur des Schreibens. 31, H. 1 (2007).

Koch, Peter/Oesterreicher, Wulf (1994): Schriftlichkeit und Sprache. In: Günther, Hartmut/Ludwig, Otto (Hrsg.) (1994): Schrift und Schriftlichkeit. Ein interdisziplinäres Handbuch internationaler Forschung. 2 Bde. Berlin/New York: de Gruyter, 1. Halbband, S. 587-604.

Krämer, Sybille (2003): Schriftbildlichkeit oder: Über eine (fast) vergessene Dimension der Schrift. In: Krämer, Sybille/Bredekamp, Horst (Hrsg.) (2003): Bild Schrift - Zahl. München: Fink, S. 157-176 (= Reihe Kulturtechnik).

Kress, Gunther/van Leeuwen, Theo (1996): Reading Images: The Grammar of Visual Design. London: Routledge (2., überarb. Auflage 2006).

Ludwig, Otto (2001): Es begann mit dem Sputnik-Schock.... Die neuere deutsche Schreibforschung. In: Praxis Deutsch Heft 170, S. 58-62.

Neef, Martin (2005): Die Graphematik des Deutschen. Tübingen: Niemeyer (= Linguistische Arbeiten 500).

Neef, Martin/Weingarten, Rüdiger (Hrsg.) (in Vorb.): Schriftlinguistik. Ein Lernund Konsultationswörterbuch mit systematischer Einleitung und englischen Übersetzungen. Bd. 5. Berlin: Mouton de Gruyter (= Wörterbücher zur Sprachund Kommunikationswissenschaft).

Roth, Kersten S./Spitzmüller, Jürgen (Hrsg.) (2007): Textdesign und Textwirkung in der massenmedialen Kommunikation. Konstanz: UVK.

Schmitz, Ulrich (2006): Schriftbildschirme. Tertiäre Schriftlichkeit im World Wide Web. In: Androutsopoulos, Jannis/Runkehl, Jens/Schlobinski, Peter/Siever, Torsten (Hrsg.) (2006): Neuere Entwicklungen in der linguistischen Internetforschung. Germanistische Linguistik, Heft 186-187, S. 184-208.

Sieber, Peter (2003): Modelle des Schreibprozesses. In: Bredel, Ursula/Günther, Hartmut/Klotz, Peter/Ossner, Jakob/Siebert-Ott, Gesa (Hrsg.) (2003): Didaktik der deutschen Sprache. 2 Bde. Paderborn: Schöningh, Bd. 1, S. 208-223.

Spitzmüller, Jürgen (2006): Typographie. In: Dürscheid, Christa (2006), S. 207-238.

Stöckl, Hartmut (2004): Typographie: Gewand und Körper des Textes - Linguistische Überlegungen zu typographischer Gestaltung. In: Zeitschrift für Angewandte Linguistik 41, S. 5-48.

Wolf, Norbert R. (2000): Texte als Bilder. In: Fix, Ulla/Wellmann, Hans (Hrsg.) (2000), S. 289-305.

Written Language and Literacy. Hrsg. v. Verhoeven, Ludo/Schreuder, Robert. Amsterdam: John Benjamins.

Zeitschrift für Sprachwissenschaft. Jubiläumsheft. Orthographie und Sprachwissenschaft. Bd. 26 (2007).

Adresse der Verfasserin:

Prof. Dr. Christa Dürscheid, Deutsches Seminar, Universität Zürich, Schönberggasse 9, CH-8001 Zürich.

E-Mail:duerscheid@ds.uzh.ch 